\title{
Recent developments in the Parton Branching approach to TMDs
}

\author{
Lissa Keersmaekers $^{a, *}$ and Sara Taheri Monfared ${ }^{b}$ \\ ${ }^{a}$ Department of Physics, University of Antwerp, \\ Groenenborgerlaan 171, 2020 Antwerpen, Belgium \\ ${ }^{b}$ Deutsches Elektronen-Synchrotron DESY, \\ Notkestraße 85, D-22607 Hamburg, Germany \\ E-mail: lissa.keersaekers@uantwerpen.be, sara.taheri.monfared@desy.de
}

The Parton Branching (PB) Method describes the evolution of transverse momentum dependent parton densities (TMDs). The obtained TMDs can be used in Monte Carlo generators to describe physical observables. We give an overview of recent developments, in particular the new application of PB TMDs in Drell-Yan + jets production, the four-flavor and five-flavor schemes for heavy quark contributions, and the inclusion of QED corrections in the PB evolution.

*** The European Physical Society Conference on High Energy Physics (EPS-HEP2021), ***

*** 26-30 July $2021 * * *$

*** Online conference, jointly organized by Universität Hamburg and the research center DESY ***

${ }^{*}$ Speaker 


\section{Introduction}

The development of transverse momentum dependent (TMD) factorization theorems ([1] and references therein) has lead to an increase of precision in predictions of observables such as the Drell-Yan (DY) transverse momentum spectrum.

The Parton Branching (PB) method [2-4] presents an angular ordered evolution for TMD parton distribution functions (TMD PDFs or TMDs), expressed in terms of real-emission splitting functions and Sudakov form factors. The PB TMDs were fitted [4] to the full HERAI+II inclusive DIS data using the XFITTER [5] framework and are available in TMDlib [6], a library for TMDs and unintegrated PDFs. These TMDs were applied to Drell Yan production [7-9].

With new software developments, such as the release of TMDlib2 [10], which includes new functionalities (e.g. the treatment of TMD uncertainties), and the newest version of the Monte Carlo event generator CASCADE [11] (CASCADE3 [12]), which includes an intial state parton shower that is fully consistent with the PB TMDs, the applications of PB TMDs will increase.

This article will give an overview of recent developments within the Parton Branching method, both in terms of new applications as new developments in the evolution.

\section{Parton Branching Evolution equations}

The Parton Branching evolution equations are given by:

$$
\begin{aligned}
\tilde{\mathcal{A}}_{a}\left(x, \boldsymbol{k}, \mu^{2}\right)= & \Delta_{a}\left(\mu^{2}\right) \tilde{\mathcal{A}}_{a}\left(x, \boldsymbol{k}, \mu_{0}^{2}\right)+\sum_{b} \int \frac{d^{2} \boldsymbol{\mu}^{\prime}}{\pi \mu^{\prime 2}} \frac{\Delta_{a}\left(\mu^{2}\right)}{\Delta_{a}\left(\mu^{\prime 2}\right)} \Theta\left(\mu^{2}-\mu^{\prime 2}\right) \Theta\left(\mu^{\prime 2}-\mu_{0}^{2}\right) \times \\
& \times \int_{x}^{z_{M}} d z P_{a b}(z) \tilde{\mathcal{A}}_{b}\left(\frac{x}{z}, \boldsymbol{k}+(1-z) \boldsymbol{\mu}^{\prime}, \mu^{\prime 2}\right),
\end{aligned}
$$

with $\tilde{\mathcal{A}}_{a}\left(x, \boldsymbol{k}, \mu^{2}\right)=x \mathcal{A}_{a}\left(x, \boldsymbol{k}, \mu^{2}\right)$ the momentum weighted TMD of flavor $a$, with longitudinal momentum fraction of the proton $x$ and $\boldsymbol{k}$ the transverse momentum, evaluated at scale $\mu, P_{a b}(z)$ the real-emission part of the DGLAP splitting functions for a splitting of parton $b$ to $a$, with $z$ the longitudinal momentum fraction and the Sudakov form factor for a parton of flavor $a$ is given by $\Delta_{a}\left(\mu^{2}\right)=\exp \left[-\sum_{b} \int_{\mu_{0}^{2}}^{\mu^{2}} \frac{d \mu^{\prime 2}}{\mu^{\prime 2}} \int_{0}^{z_{M}} d z z P_{b a}^{c o l}\left(z, \mu^{\prime 2}\right)\right]$. Angular ordering can enter the evolution through three aspects: i) the relation between the evolution scale $\mu^{\prime}$ and the transverse momentum of the emitted parton $\boldsymbol{q}$ : $(1-z) \mu^{\prime}=|\boldsymbol{q}|$, which is embodied in all PB TMDs, ii) the scale of the strong coupling $\alpha_{s}\left(\boldsymbol{q}^{2}\right)$ which is present in the fitted PB-NLO-HERAI+II-2018-set2, but not in PBNLO-HERAI+II-2018-set1, which uses $\alpha_{s}\left(\mu^{\prime 2}\right)$, iii) the dynamical (i.e. dependent on the evolution scale) soft-gluon resolution scale $z_{M}=1-q_{0} / \mu^{\prime}$, with $q_{0}$ the minimal transverse momentum of the emitted parton. The resolution scale seperates resolvable from non-resolvable branchings. The effects of the dynamical resolution scale have been studied in [8].

\section{Multijet-merging}

Studies of TMD effects have been so far mostly used on low $p_{\perp}$-spectra of inclusive observables. However, the authors of [13] realized that the large transverse momentum tails of TMDs, that arise naturally due to the renormalization-group evolution are used to describe multi-jet final states. A 

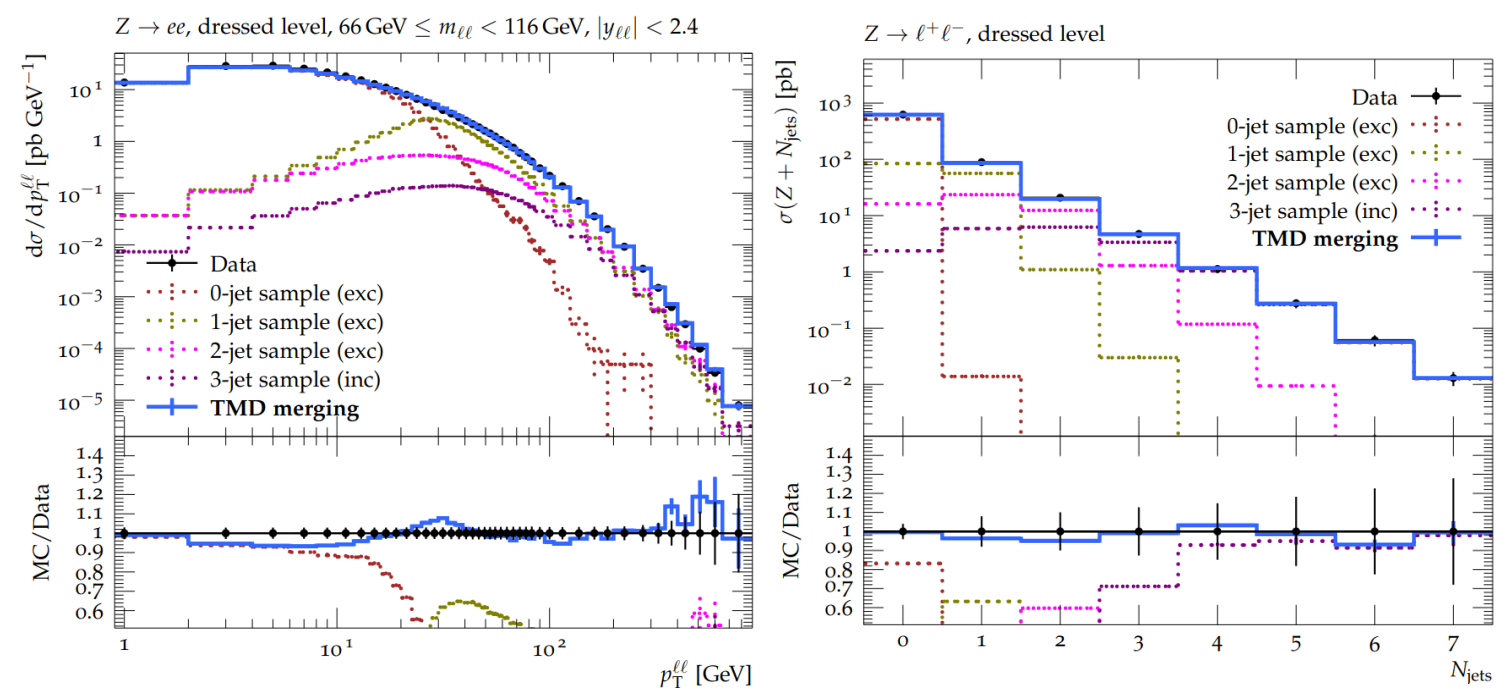

Figure 1: Predictions obtained with TMD merging for the production of a Z-boson in association with jets. Left: Z-boson $p_{\perp}$-spectrum; Right: Jet multiplicity. Figures from [13].

new "TMD merging" algorithm has been developed in [13], which extends the "MLM merging" procedure to include TMD initial state evolution. Compared to standard MLM, this method reduces systematical uncertainties and improves the description of higher-order emissions beyond the maximum parton multiplicity of the matrix element calculations.

In figure 1 , their prediction of the Z-boson $p_{\perp}$-spectrum and jet-multiplicity is shown. For these results the PB-NLO-HERAI+II-2018-set2 TMDs where used. The TMD merging algorithm describes the whole Z-boson $p_{\perp}$-range very well. The description of Jet-Multiplicity is remarkable, especially for multiplicities that are higher than the jet multiplicity of the matrix element, which is three. One can expect that the effects studied in their work will become even more important at future collider experiments, since TMD broadening grows with the evolution scale.

\section{Photon TMD}

To obtain the same accuracy as current experimental programs, electroweak corrections should be applied to the before purely QCD evolution of the PB method. The most notable change in the QED corrected evolution of parton distributions is the presence of the photon density. We determined both collinear and TMD photon densities with PB method [14].

At high mass Drell-Yan (DY) production, contributions from photon-photon scattering into lepton pairs play a role. The collinear NLO QED PDFs describe well the measured dilepton mass spectrum at LHC center-of-mass energies [15]. As shown in Fig.2.a, the small contribution from Photon-initiated (PI) lepton production is also determined. The photon TMD has been used to predict the transverse momentum spectrum of DY lepton-pair production at very high masses (Fig 2.b). 


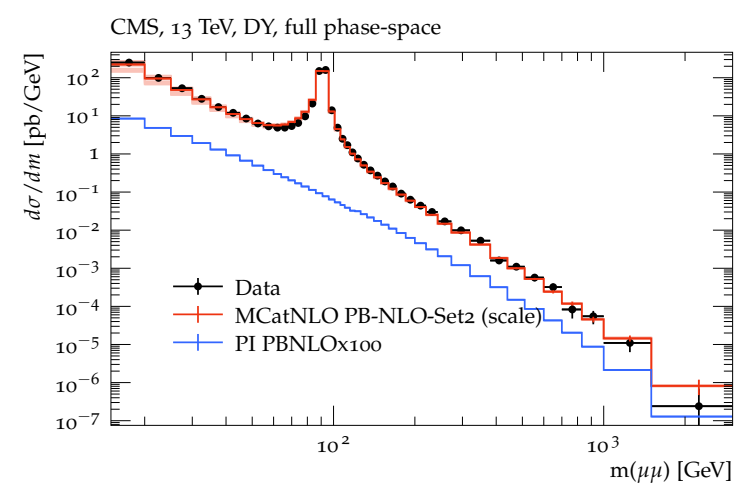

$\mathrm{a}$

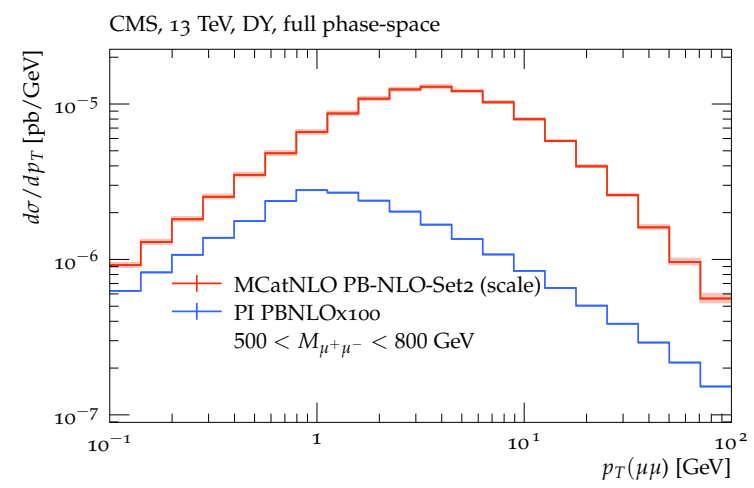

$\mathrm{b}$

Figure 2: Standard DY and photon induced mass distribution (a) and transverse momentum spectra (b) based on collinear and TMD QED PDFs

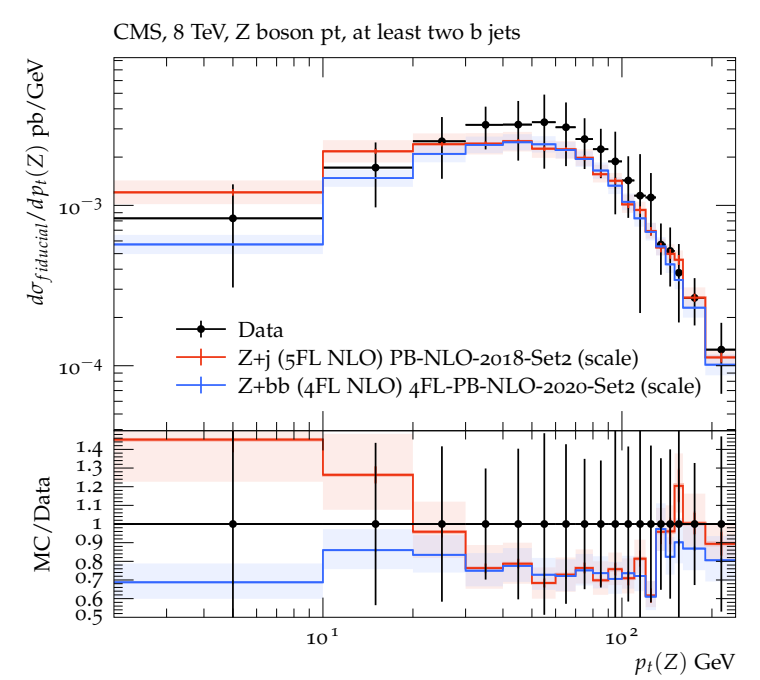

a

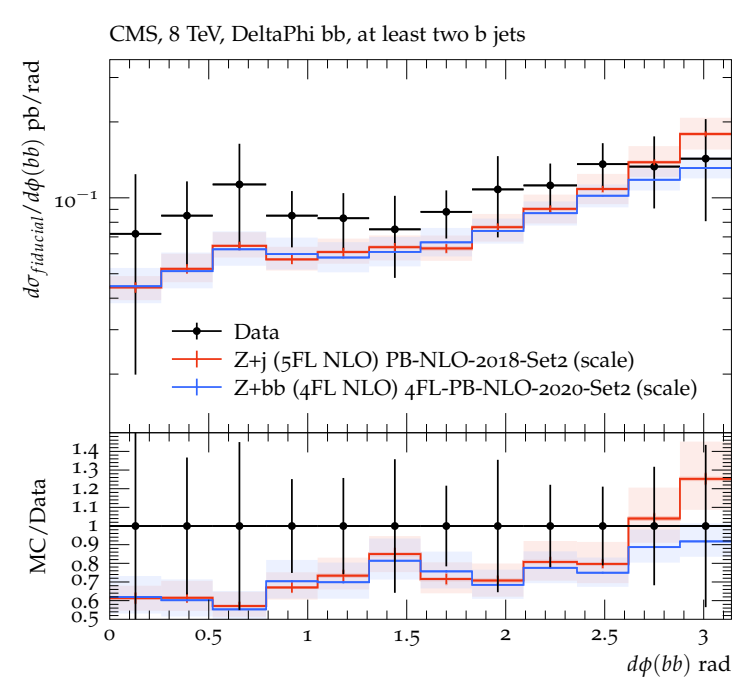

$\mathrm{b}$

Figure 3: Differential cross section for $Z+\geq 2 b$ jets production as a function transverse momentum of the $Z$ boson $p_{t}$ (a) and the azimuthal angular separation $\Delta \phi_{b b}$ between the directions of the two $b$ jets in the transverse plane (b). Shown are the predictions obtained in the 4FLVN- and 5FLVN- schemes.

\section{Four- and Five-flavor schemes}

The first set of NLO collinear and TMD parton densities in four-flavor-variable-number (4FLN) scheme within the PB approach is determined [16]. The 4FLVN and five-flavor-variable-number (5FLVN) PB-TMD distributions [4] were applied to predict $Z+b \bar{b}$ tagged jet production at LHC energies. In Fig. 3, we show the predictions obtained within both schemes, which are in very good agreement with the measurements.

The completely different configurations of heavy flavor collinear and TMD PDFs and the corresponding initial TMD parton shower in the 4FLVN and 5FLVN schemes allow for a precise investigation of the evolution of the PB-TMD PDFs as well as the PB-TMD parton shower. 


\section{Conclusion}

The Parton Branching method has already had several successes last years, especially in the description of the low- $p_{\perp}$ spectrum of the Drell Yan process at both high and low energies[7-9]. With the new developments, the range of applications increase, due to e.g. the new TMD merging method, which allows a description of the whole $p_{\perp}$-spectrum of the Drell Yan process and an accurate description of jets, even at high mltiplicity.

The first PB TMD PDF set within the four-flavor schemes along with the already existing sets in the five-flavor scheme opens the further investigation of PB evolution and PB TMD showers.

Other developments of the PB TMDs will lead to an increase in precision or an extension of the kinematical range. The first inclusion QED effects in the Parton Branching method, including the first photon TMD within the method is obtained. The inclusion of TMD splitting functions [17-22] is underway [23], and it is a first step towards a Monte Carlo that incorporates small- $x$ dynamics.

\section{Acknowledgements}

We thank F. Hautmann, M. Hentschinski, H. Jung, A. Bermudez Martinez, A. Kusina, K. Kutak and A. Lelek for collaboration and discussion. STM thanks the Humboldt Foundation for the Georg Forster research fellowship.

\section{References}

[1] R. Angeles-Martinez et al., Transverse Momentum Dependent (TMD) parton distribution functions: status and prospects, Acta Phys. Polon. B 46 (2015) 2501 [1507.05267].

[2] F. Hautmann, H. Jung, A. Lelek, V. Radescu and R. Žlebčík, Soft-gluon resolution scale in qcd evolution equations, Physics Letters B 772 (2017) 446 [1704 . 01757].

[3] F. Hautmann, H. Jung, A. Lelek, V. Radescu and R. Žlebčík, Collinear and tmd quark and gluon densities from parton branching solution of qcd evolution equations, Journal of High Energy Physics 2018 (2018) 70 [1708.03279].

[4] A.B. Martinez, P. Connor, H. Jung, A. Lelek, R. Žlebčík, F. Hautmann et al., Collinear and tmd parton densities from fits to precision dis measurements in the parton branching method, Phys. Rev. D 99 (2019) 074008 [1804 . 11152].

[5] S. Alekhin, O. Behnke, P. Belov, S. Borroni, M. Botje, D. Britzger et al., Herafitter, The European Physical Journal C 75 (2015) 304 [1410 .4412].

[6] F. Hautmann, H. Jung, M. Krämer, P.J. Mulders, E.R. Nocera, T.C. Rogers et al., Tmdlib and tmdplotter: library and plotting tools for transverse-momentum-dependent parton distributions, The European Physical Journal C 74 (2014) 3220 [1408 . 3015].

[7] A. Bermudez Martinez, P.L.S. Connor, D. Dominguez Damiani, L.I. Estevez Banos, F. Hautmann, H. Jung et al., Production of z bosons in the parton branching method, Phys. Rev. D 100 (2019) 074027 [1906. 00919]. 
[8] F. Hautmann, L. Keersmaekers, A. Lelek and A. van Kampen, Dynamical resolution scale in transverse momentum distributions at the lhc, Nuclear Physics B 949 (2019) 114795 [1908.08524].

[9] A.B. Martinez, P.L.S. Connor, D.D. Damiani, L.I.E. Banos, F. Hautmann, H. Jung et al., The transverse momentum spectrum of low mass drell-yan production at next-to-leading order in the parton branching method, The European Physical Journal C 80 (2020) 598 [2001.06488].

[10] N.A. Abdulov, A. Bacchetta, S. Baranov, A. Bermudez Martinez, V. Bertone, C. Bissolotti et al., Tmdlib2 and tmdplotter: a platform for 3 d hadron structure studies, The European Physical Journal C 81 (2021) 752 [2103.09741].

[11] H. Jung, S. Baranov, M. Deak, A. Grebenyuk, F. Hautmann, M. Hentschinski et al., The ccfm monte carlo generator cascade version 2.2.03, The European Physical Journal C 70 (2010) 1237.

[12] S. Baranov, A. Bermudez Martinez, L.I. Estevez Banos, F. Guzman, F. Hautmann, H. Jung et al., Cascade3 a monte carlo event generator based on tmds, The European Physical Journal C 81 (2021) 425 [2101.10221].

[13] A.B. Martinez, F. Hautmann and M.L. Mangano, TMD Evolution and Multi-Jet Merging, 2107.01224.

[14] H. Jung, S. Taheri Monfared and T. Wening, Determination of collinear and TMD photon densities using the Parton Branching method, Phys. Lett. B 817 (2021) 136299 [2102.01494].

[15] A.M. Sirunyan, A. Tumasyan, W. Adam, F. Ambrogi, T. Bergauer, J. Brandstetter et al., Measurements of differential $z$ boson production cross sections in proton-proton collisions at $\sqrt{s}=13$ tev, Journal of High Energy Physics 2019 (2019) 61 [1909.04133].

[16] H. Jung and S.T. Monfared, Tmd parton densities and corresponding parton showers: the advantage of four-and five-flavour schemes, 2106.09791.

[17] S. Catani and F. Hautmann, High-energy factorization and small-x deep inelastic scattering beyond leading order, Nuclear Physics B 427 (1994) 475.

[18] F. Hautmann, M. Hentschinski and H. Jung, Forward Z-boson production and the unintegrated sea quark density, Nucl. Phys. B 865 (2012) 54 [1205 . 1759].

[19] O. Gituliar, M. Hentschinski and K. Kutak, Transverse-momentum-dependent quark splitting functions in kt-factorization: real contributions, Journal of High Energy Physics 2016 (2016) 181 [1511.08439].

[20] M. Hentschinski, A. Kusina and K. Kutak, Transverse momentum dependent splitting functions at work: quark-to-gluon splitting, Phys. Rev. D 94 (2016) 114013 [1607.01507]. 
[21] M. Hentschinski, A. Kusina, K. Kutak and M. Serino, Tmd splitting functions in $k_{t}$ factorization: the real contribution to the gluon-to-gluon splitting, The European Physical Journal C 78 (2018) 174 [1711.04587].

[22] M. Hentschinski, Transverse Momentum Dependent Gluon Distribution within High Energy Factorization at Next-to-Leading Order, 2107.06203.

[23] L. Keersmaekers, Implementing transverse momentum dependent splitting functions in parton branching evolution equations, 2109.07326. 